\title{
MirrorForge: Rapid Prototyping of Complex Mirrors for Camera and Projector Systems
}

\author{
Christopher Getschmann \\ cget@cs.aau.dk \\ Aalborg University \\ Aalborg, Denmark
}

\author{
Everett M. Mthunzi \\ mondliwethu.everett.mthunzi@uni- \\ weimar.de \\ Bauhaus-Universität Weimar \\ Weimar, Germany
}

\author{
Florian Echtler \\ floech@cs.aau.dk \\ Aalborg University \\ Aalborg, Denmark
}

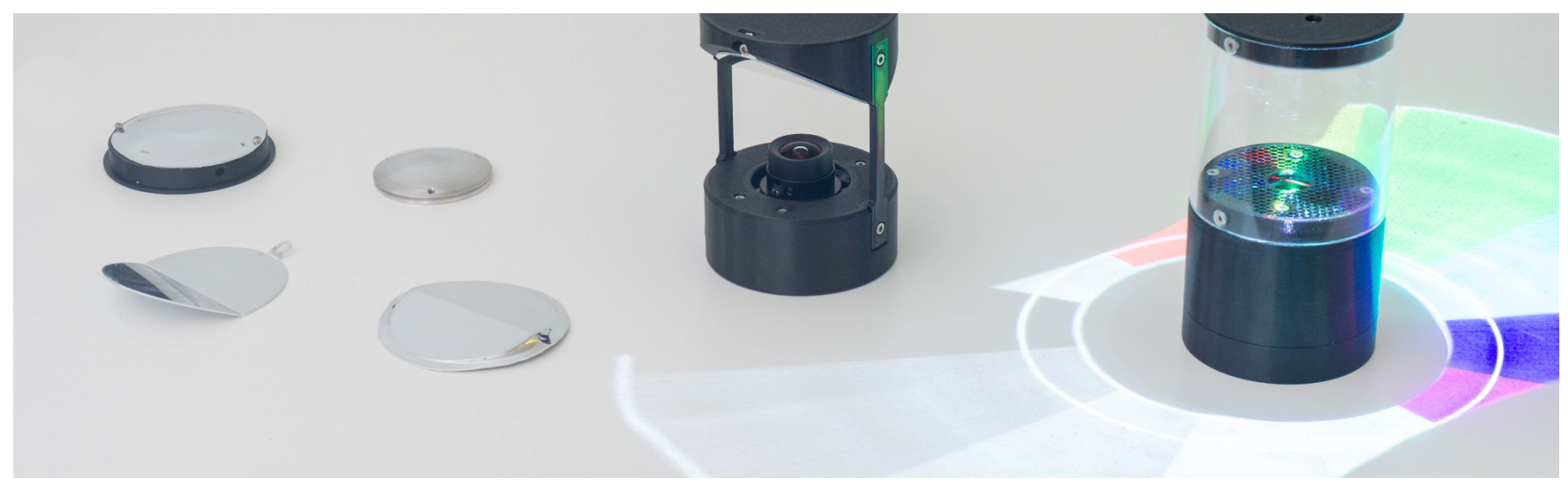

Figure 1: To decrease size or increase the field of view, mirrors are used to optimize the optical path geometry. This is often the case for small and mobile prototypes such as tangibles. Laminated polystyrene mirrors mounted on fixtures are suitable for usage with cameras and projectors alike.

\begin{abstract}
From small tangibles to large tabletops, mirrors with complex geometries can be invaluable tools to reflect or form light for cameras and projectors. While many fabrication techniques are available for prototyping physical or electronic components, creating mirrors requires manual computation and industrial manufacturing equipment and is therefore considerably slower and more expensive. We propose a technique for fabricating mirror surfaces based on thermoplastics sheets with a laminated metallization layer and 3D-printed fixtures for bending or vacuum forming. Given our toolchain, a simulation of mirrors for cameras and projectors by rendering the $\mathrm{CAD}$ model is possible and allows to fabricate and evaluate design iterations quickly, making the process reasonably accessible for research. Finally, we show two prototypes for tangible interfaces based on our mirrors for projection and camera-based interaction, discussing advantages and limitations.
\end{abstract}

Permission to make digital or hard copies of part or all of this work for personal or classroom use is granted without fee provided that copies are not made or distributed for profit or commercial advantage and that copies bear this notice and the full citation on the first page. Copyrights for third-party components of this work must be honored

For all other uses, contact the owner/author(s).

TEI '22, February 13-16, 2022, Daejeon, Republic of Korea

(c) 2022 Copyright held by the owner/author(s).

ACM ISBN 978-1-4503-9147-4/22/02.

https://doi.org/10.1145/3490149.3501329

\section{CCS CONCEPTS}

- Human-centered computing $\rightarrow$ Ubiquitous and mobile computing systems and tools; Systems and tools for interaction design; Visualization systems and tools.

\section{KEYWORDS}

mirror, projection, rapid prototyping, 3D printing, thermoforming

\section{ACM Reference Format:}

Christopher Getschmann, Everett M. Mthunzi, and Florian Echtler. 2022. MirrorForge: Rapid Prototyping of Complex Mirrors for Camera and Projector Systems. In Sixteenth International Conference on Tangible, Embedded, and Embodied Interaction (TEI '22), February 13-16, 2022, Daejeon, Republic of Korea. ACM, New York, NY, USA, 7 pages. https://doi.org/10.1145/3490149. 3501329

\section{INTRODUCTION}

Mirrors are used in a wide variety of prototypes built for HumanComputer Interaction (HCI) research to redirect light for camera and projector applications (see fig. 1). In general, non-planar mirrors (see fig. 2) can enable otherwise infeasible applications and will result in different cost and complexity considerations for fabrication and use. Simple, planar mirrors are available in high quality as offthe-shelf components and are used for many projection applications in stationary $[5,8,13,16,18]$ and mobile prototypes [19]. Omnidirectional cameras (i.e. cameras facing a rotation-symmetric mirror) have a significantly more complex geometry to capture a distorted 360-degree view. Researchers using these either require expensive 


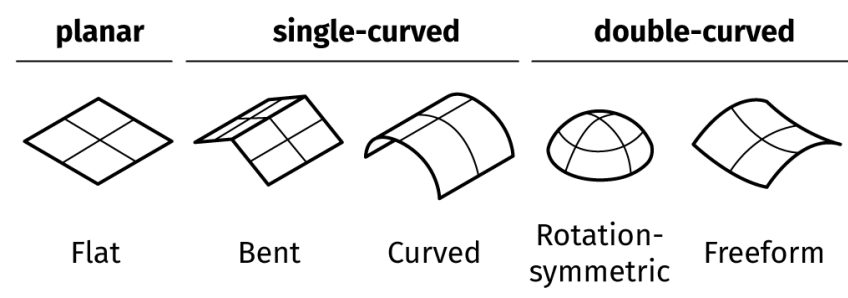

Figure 2: Common geometries of surfaces. Single-curved geometries can be created by bending thin materials (folding a sheet of paper without tearing it). Double-curved surfaces require a controlled deformation (stretching or compressing) or subtraction of the material and are only suitable for significantly more complex manufacturing techniques.
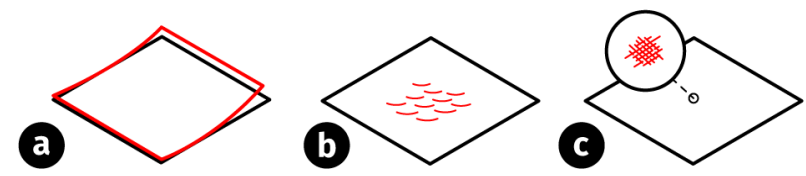

Figure 3: Common issues with mirror surface parts: a) Overall dimensional accuracy of the part (deviations in flatness or specified curvature). Visible as distortion in the reflected image. b) Surface deformations visible on the mirror such as dents (example: "orange peel effect"). Visible as local distortions and illumination differences. c) Surface roughness on the nanometer scale, visible as fogging or blurring.

external manufacturing by computer-controlled machining on a lathe [7] or make use of randomly available and often ill-fitting offthe-shelf parts [1]. When larger mirrors are required, especially for projection, fabrication by thermoforming and aluminium coating is the most viable option. These mirrors can either be repurposed domes intended for indoor surveillance [2] or specifically manufactured parts based on the researchers' curvature calculations [12]. In general, prototypes for HCI applications often make use of rapid-prototyping techniques such as $3 \mathrm{D}$ printing or laser cutting. This allows fast and cheap manufacturing of small batches of highly customized parts, reducing the need to rely on off-the-shelf components. However, each of these techniques imposes a multitude of process-related constraints such as kerf (material removal along the laser line) for laser cutters or support structures for 3D printing. Common to all rapid-prototyping methods are imperfections or reduced surface finish. This makes these methods unsuitable for parts that require high dimensional accuracy or smooth surfaces.

To achieve a mirror surface for optical applications, the material needs to satisfy accuracy and maximum roughness requirements on different scales (see fig. 3). When planar or curved mirrors are required, three common manufacturing techniques are available:

- Milling or turning metal on a CNC lathe, followed by polishing so the surface of the metal itself becomes a mirror.

- Pouring or grinding glass to create a flat surface, followed by coating the surface with a reflective material.

- Thermoforming, followed by coating with a reflective material.
Thermoforming is the fabrication method of deforming heated plastic sheets with pressurized air or molds and can be classified both as mass manufacturing as well as a prototyping technique. Thermoforming, or vacuum forming if a vacuum is used to pull the material over the mold, has the advantage of creating very smooth surfaces without additional processing steps due to the surface tension of the molten plastic. However, all of these techniques either require expensive tooling for turning and polishing the raw material or a second process step is necessary for electroplating or sputtering in a vacuum chamber. This makes the process of fabricating a mirror slow and costly, often involving external manufacturing with equipment not available in most research labs. Running multiple iterations to refine the shape is an expensive and time-consuming issue.

Thus, prior to fabrication, mirror geometries require evaluation, but there are very few tools available in between back-of-a-napkin sketches or professional optical modeling software packages. While calculating $2 \mathrm{~d}$ rays is sufficient to analyze angle, magnification factor, and distortion, the usage scenario for the required mirror may be considerably more complex. At the same time is optical simulation software often out of reach or unsuitable for the issue at hand. By rendering the mirror with a raytracer, we can visualize the effect of enclosure elements or different mirror positions easily in an environment familiar to many researchers.

We contribute a technique to prototype functional mirrors with both simple and complex geometries in a fast and inexpensive way using basic tools such as 3D printers and improvised vacuum formers. With our software, we support the different stages of designing and fabricating mirrors. This includes:

- A frontend for simulating mirrors for projector and camera applications based on Blender and LuxCoreRender engine.

- A process to rapidly prototype complex mirror surfaces using laminated thermoplastic sheets and 3D-printed molds or fixtures.

- A comparison of suitable materials for thermoforming mirror prototypes.

- Two application prototypes which demonstrate how rapidprototyping of mirrors can be leveraged for designing, evaluating, and building high-fidelity interaction prototypes.

\section{RELATED WORK}

Fabrication techniques using thermoplastics

Among common fabrication techniques thermoforming is a simple and inexpensive way of creating curved parts. Schüller et al. [15] simulate the expected distortions during the thermoforming process. This allows to control the surface color of the finished shape by printing a distorted image on the flat stock material. Interactive control of the thermoforming process is demonstrated by FormFab [11] (using a steerable heat source) and ProtoMold [21] (using a steerable mold), however, these approaches are only suitable for freeform prototypes without precise dimensional requirements.

Another approach for digital fabrication with thermoplastic sheets is presented in LaserOrigami [10], where an intentionally defocused laser cutter is used to selectively melt bending lines in the material after the focused laser has been used to cut the 
unfolded shape. This allows the automated creation of piecewiseplanar shapes from thermoplastics, similar to sheet-metal bending techniques. An innovative use for metallized thermoplastics has been presented by Wojakowski et al. [20] who use a laser cutter to selectively remove the metallization layer prior to thermoforming, thereby embedding simple circuits directly into the final shape.

\section{Mirror calculation and fabrication}

To the best of our knowledge, there is little research on prototyping techniques to substitute mirror surfaces. The rapid prototyping approach presented by Vaidya et al. [17] allows to fabricate complex mirror geometries with low-cost 3D-printing methods but requires additional smoothing and aluminium coating steps.

The majority of work concerning mirrors is conducted in order to calculate curvatures suitable for different applications of omnidirectional cameras and exclusively makes use of $\mathrm{CNC}$-controlled lathes for fabrication (e.g. [3, 7]) with optimized toolpaths and specialized diamond tooling to achieve a mirror finish.

Piovarči et al. [14] compute and fabricate micro-mirror arrays for directional projection screens by $\mathrm{CNC}$ milling flat aluminium plates, followed by manual polishing. Müller et al. describe their application-specific approach to calculate and fabricate a mirror for BaseLase [12], an omnidirectional laser projector. BaseLase uses thermoforming to fabricate the mirror geometry but requires subsequent industrial metal sputtering for the reflective surface layer.

\section{Mirror applications}

In the field of human-computer interaction, most research prototypes that make use of mirrors rely on commonly available parts, e.g. planar surface mirrors in many interactive surfaces such as Wilson's PlayAnywhere [18], HoloDesk by Hilliges et al. [5], or more complex dual-mirror setups to shorten the optical path as in [4]. Liang et al. [9] use fingertip-sized bent mirrors as tangibles to augment printed paper.

For specific camera-based prototypes such as MeCap [1] or projector applications such as Hölscher et al. [6], off-the-shelf hemispherical mirrors are sometimes repurposed, although their exact geometry is usually unknown beforehand and may require compensation in software.

\section{MIRROR DESIGN AND SIMULATION}

Design and simulation of mirrors will be discussed shortly before suitable materials are compared and fabrication techniques for bent, curved, and freeform mirrors are described.

Our fabrication technique is supported by custom software: our MirrorForge plugin for the CAD/CAM software Fusion360 from Autodesk. While Fusion 360 offers tools for parametric design and rendering, neither of these tools is suitable for mirrors. The MirrorForge plugin allows the import of mirror curvatures and exports scenes including mirror objects for a physically correct simulation. This way our tool integrates nicely with the prototyping workflow, filling gaps where state-of-the-art CAD software is insufficient for the task of prototyping mirrors.

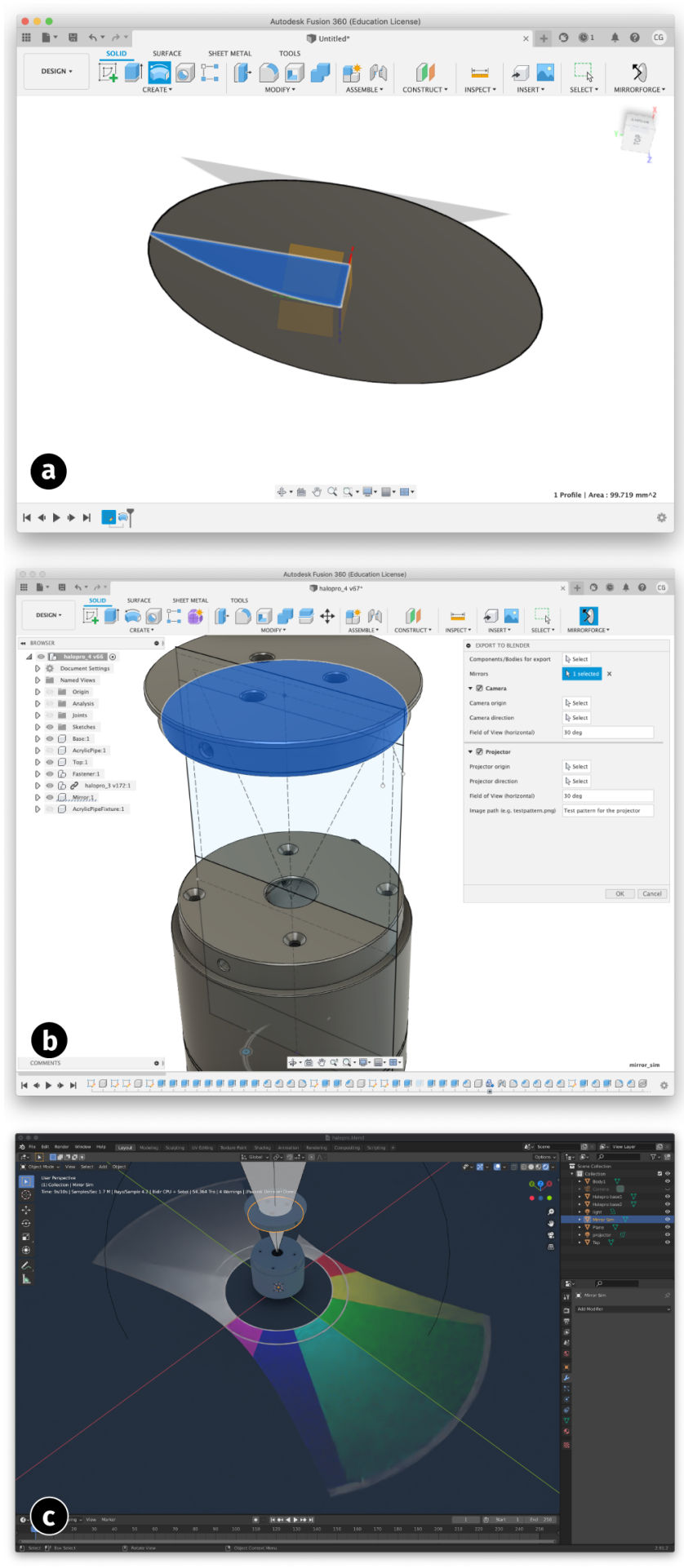

Figure 4: a) The MirrorForge plugin allows importing a mirror curvature as points from CSV files. The $2 \mathrm{D}$ shape can be extruded to a $3 \mathrm{D}$ object which should be fabricated as a mirror. b) The plugin allows the export of the whole design, selecting geometries that should be rendered as mirror surfaces and specifying camera or projector positions and angles. c) The MirrorForge Blender integration creates a scene for rendering using the exported models. 


\section{Mirror design}

Flat and bent mirrors can easily be designed with standard tools in parametric CAD software such as Fusion360. Curved or rotationsymmetric shapes are more complicated. Our MirrorForge plugin allows importing precalculated curves from CSV files, representing them as connected lines in a technical drawing in Fusion360. This has a considerably lower computational footprint than splines, the default tool for curved geometries in Fusion360. The calculation of a suitable curvature is not within the scope of this paper, but once a curvature has been calculated and is imported, the resulting mirror needs to be evaluated.

\section{Simulation}

Evaluation of mirror designs is usually done analytically by computing the maximum and minimum rays and their angles of incidence in a 2D plane. This is sufficient for scenarios such as rotationsymmetric mirrors in omnidirectional cameras. When dealing with more complex scenarios, non-symmetric mirrors, or additional elements like fixtures and enclosures which may partially block the mirror, 2D analysis may be insufficient. The MirrorForge plugin makes it possible to transfer models directly from CAD to simulation. Interactive rendering is realized by the LuxCore ${ }^{1}$ engine in bidirectional mode with Blender ${ }^{2}$ as the frontend for interactive manipulation of the rendered scene. The LuxCore rendering engine is necessary to allow raytracing of both cameras and projectors since Blender's engines do not render reflected rays of projectors. The 3D data of objects and mirror surfaces are passed on from CAD to simulation in polygon meshes (STL files) and thus suffers from reduced precision. Using meshes introduces artifacts in the reflected image due to the difference in angle for reflected rays between adjacent (planar) polygon faces. To mitigate these issues, mesh data needs to be smoothed by remeshing prior to raytracing (see fig. 5). Additional smoothing during shading can improve visual quality but will introduce a geometrical error in mirror regions with low angles of incidence.

\section{MIRROR FABRICATION}

\section{Materials}

Mirrors fabricated as prototypes have considerably lower quality requirements than a precision-manufactured optical component, but the combination of several fabrication steps is necessary to make the process faster and less expensive. By avoiding an additional polishing or coating process step the fabrication time of a mirror could be drastically reduced. However, this is only possible if suitable pre-coated reflective materials can be used. We selected and evaluated four widely accessible materials for their specular reflection quality (see table 1 ).

Raw materials in their flat state are compared visually by reflecting a laser projector off the surface and comparing the reflected image (see fig. 6). Visual inspection is sufficient for the quality requirements of prototyped mirrors (see fig. 7). Comparison of the reflected image reveals potential issues with the materials at all

\footnotetext{
${ }^{1}$ https://luxcorerender.org/

${ }^{2}$ https://blender.org/
}

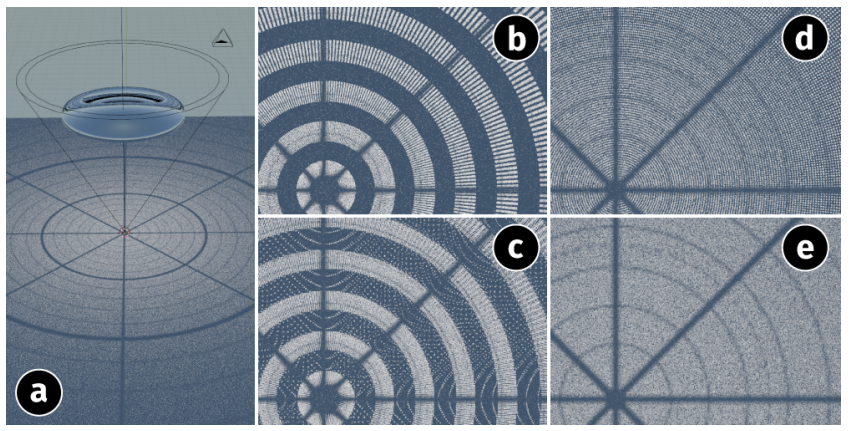

Figure 5: The polygon faces representing the curved surface result in errors in the reflected image. a) Setup projecting a test pattern image against a spherical mirror b) Reflection from a meshed body exported directly from CAD (Fusion 360 at quality setting 'high'). c) reflection after remeshing applied by Blender d) reflection of a meshed body with external remeshing applied before import. e) Applying smooth shading in Blender during raytracing (not suitable for all geometries).

\begin{tabular}{l|r} 
Stock material & thickness [mm] \\
\hline \hline Metallized acrylic glass & 3.00 \\
Polymethyl methacrylate (PMMA) & 1.00 \\
\hline $\begin{array}{l}\text { Laminated PS sheet } \\
\text { Polystyrene (PS) }\end{array}$ & 0.15 \\
\hline $\begin{array}{l}\text { Coated foil } \\
\text { Polyvinyl chloride (PVC), aluminium (Al) }\end{array}$ & 0.50 \\
\hline $\begin{array}{l}\text { PETG sheet, Rust-Oleum Mirror spray } \\
\text { Polyethylene terephthalate glycol (PETG) }\end{array}$ &
\end{tabular}

Table 1: Pre-coated materials evaluated for reflection quality.

three scales (see fig. 3). Spray-coated PETG creates a blurred and low-intensity reflection, considered unusable as a material. Coated PMMA exhibits the highest amount of flatness while reflecting visible micro-fractures. Laminated PS requires a fixture to be flat but shows an overall uniform reflection with only small circular variations in brightness. Note that laminated PS is the only material showing color banding, apparently caused by a difference in refraction among wavelengths. Aluminium coated foil shows strong distortions and is considered unusable. Reference material is an off-the-shelf mirror (borosilicate glass with silver coating on the backside) with no visible issues in the reflection.

The reflected image of both PMMA and PS would be suitable for prototyping mirrors, but PS is considerably easier to bend and thermoform than acrylic glass and should be preferred. Metallized polystyrene is a widely available material for industrial applications as well as hobbyists in the arts-and-crafts sector. Although it is advertised as "not suitable for vacuum forming" or "suitable for shallow vacuum forming" it does perform well for the intended use. If no PS is available, PVC or PETG sheets of $0.5-1 \mathrm{~mm}$ thickness with a laminated metallization layer may perform similarly. 


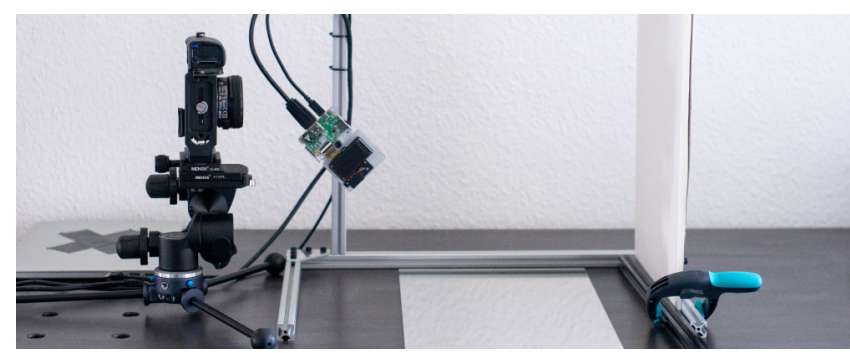

Figure 6: Setup for visual inspection. Light source: Ultimems HD301A1-H2 laser projector, projection plane: paper.

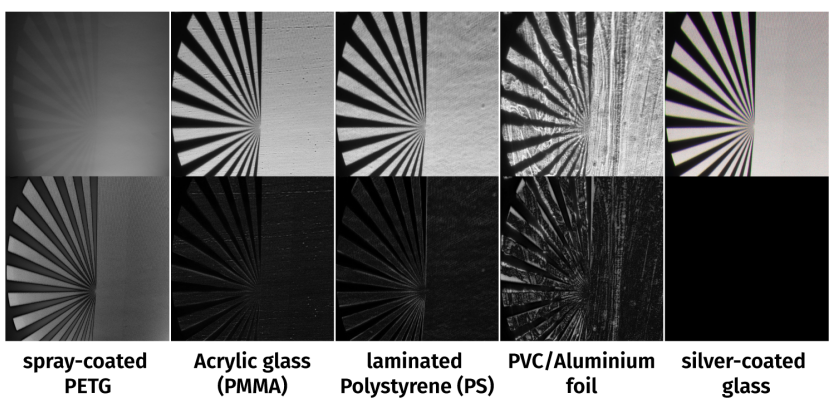

Figure 7: Reflection of each tested material at a 45-degree angle. Reference material is glass with silver coating on the backside. Upper row is the photo of the reflected image (BW image source, RGB photo of reflection), lower row is pixelwise difference compared to the reflection of silver-coated glass as reference (less is better).

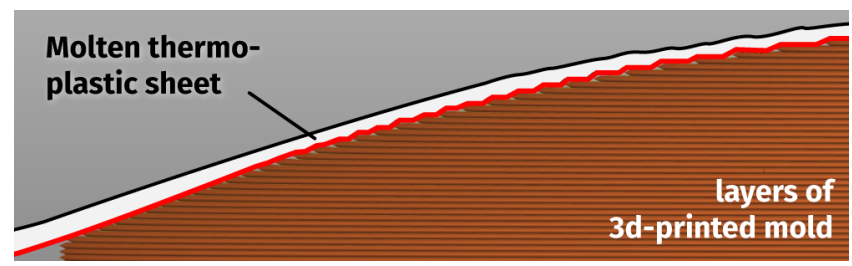

Figure 8: Cross section of a 3D-printed mold: rotationsymmetric mirror of $65 \mathrm{~mm}$ diameter, sliced at $0.2 \mathrm{~mm}$ layer height. The layer lines are clearly visible and will imprint on the mirror surface once the molten thermoplastic has cooled.

\section{Thermoforming}

To ensure dimensional accuracy a fixture is required to hold the formed mirror sheets. This is true for bent and curved sheets, as well as thermoformed parts. When an improvised vacuum former and a heat gun is used, laminated PS may be unevenly heated and tends to warp when cooling, using a sufficient fixture is then mandatory. 3D-printed fixtures for bent or curved parts and molds for thermoformed mirrors satisfy both requirements to guarantee dimensional accuracy of the part as well as allow fabrication at the same time (see fig. 10). To create a mold from a mirror geometry

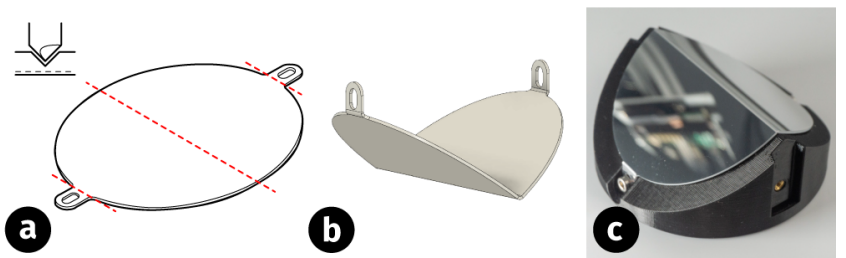

Figure 9: Toolpaths for carving and cutting on a CNC (a). Precarved bending lines are a simple way to create precise bends for manual handling (b). To align the mirror at accurate angles a 3D-printed fixture is used in the prototype (c).

in CAD, the models' mirror surface needs to be offset by the thickness of the sheet material (1mm in the case of laminated PS) and additional features such as screw holes or cavities for fasteners can be added. When relying on Fused Deposition Modeling (FDM) 3D printers to create the part, factory default print settings will result in unusable molds. Due to the layer-by-layer deposition of FDM 3D printers, shallow curvatures introduce a noticeable error and layer lines will be visible on the mirror (see fig. 8). An additional issue is posed by the heated thermoplastic used for vacuum forming which transfers heat to the printed mold and may melt the surface. If this occurs, partial melting will emboss the structure of the infill on the distorted mirror surface. The glass temperature of PS $\left(100^{\circ} \mathrm{C}\right)$ is above the glass temperature of the most common 3D-printing materials such as Polylactide (PLA, $\left.45-65^{\circ} \mathrm{C}\right)$, PETG $\left(67-81^{\circ} \mathrm{C}\right)$, or Acrylonitrile butadiene styrene (ABS, $\left.85-100{ }^{\circ} \mathrm{C}\right)$. The effect of heat transfer can be mitigated by heating the thermoplastic only slightly above its glass temperature (at the expense of higher viscosity and thus increased curvature along ridges in the vacuum-formed part) or by increasing the density of the printed mold. Recommended printing parameters for the mold: PETG, ABS, or high-temperature copolymer filament/resin, layer height lower than $0.05-0.08 \mathrm{~mm}$, $1 \mathrm{~mm}$ minimum wall thickness. If larger layer heights are chosen, the mold needs to be manually sanded or treated with a filler and primer. To permanently bond the mold to the thermoformed part, either a thin layer of heat-resistant glue needs to be sprayed on the mold or embedded screw holes for fasteners are required (see fig. $10)$.

\section{Bending}

If multiple flat surfaces or surfaces with a single curvature are required, bending is a simpler approach. Sheet metal bending is a well-established fabrication technique and laminated PS sheets can be handled similarly. This allows to make use of existing CAD tools to define bents at specified angles. These bending lines can be pre-carved with $\mathrm{V}$-shaped chamfering endmills on a CNC mill or by hand prior to cutting the part out of the sheet (see fig. 9). Toolpaths can be generated directly in Fusion360 alongside the MirrorForge plugin. Manual cutting making use of the technical drawing as a template is possible as well. 


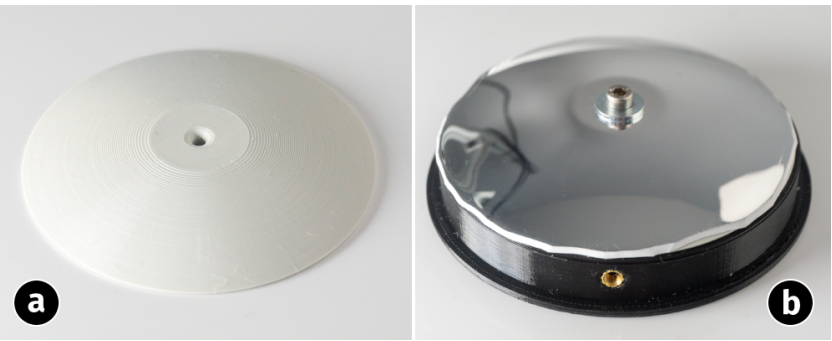

Figure 10: a) 3D-printed thermoforming mold. Printed at $0.08 \mathrm{~mm}$ layer height. The central hole improves suction during vacuum forming and is used as a screw hole for fastening the mirror. [Printed on a common FDM printer (Prusa Mini) using a $0.25 \mathrm{~mm}$ nozzle] b) The formed mirror is fixed to the mold, excess material is removed and the mold is screwed to an enclosure element. Permanently bonding mold and fixture guarantees dimensional accuracy and allows precise alignment.
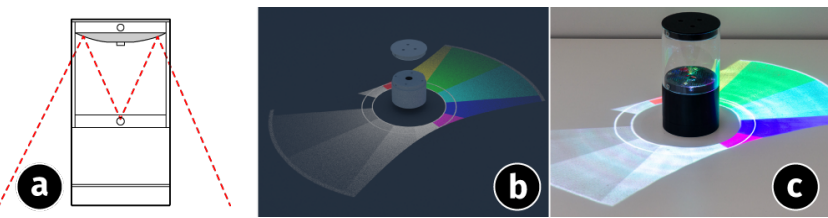

Figure 11: a) A spherical mirror is placed at the end of an acrylic pipe. The mirror is vacuum formed and fixed to the 3D-printed mold with a screw in a printed thread. The screw sits in the central dead region of the mirror. b) Rendering of the mirror to simulate the projected image prior to vacuum forming. c) Manufactured prototype of the circular tangible projector.

\section{APPLICATION EXAMPLES}

The possible applications and advantages of mirrors manufactured from PS sheets can be shown with two examples from the domain of tangible interaction prototypes:

\section{Circular Tangible Projector}

The tangible projector (see fig. 11) is fully self-contained, integrating a laser projector (Ultimems HD301A1-H2), processor, and battery in the base. A rotation-symmetric spherical mirror (see fig. 10) magnifies and reflects the projection back onto the table surface, spanning a total of $50 \mathrm{~cm}$ in diameter. Note that we are using a laser projector, thus the projected image is always in focus. This relieves us from computing a focal plane and suitable focus distance. The simulation won't account for that and these calculations would have to be done manually. The increased projection area allows the mobile projector to be used in conjunction with other tangibles, enabling interaction scenarios like a fragmented, self-projecting tabletop. The curvature of the mirror results in the desired increased projection size but does at the same time magnify the effect of imperfections in the mirror surface (see fig. 1). A loss of sharpness and isolated local distortions of $1-3 \mathrm{~mm}$ size are visible issues.
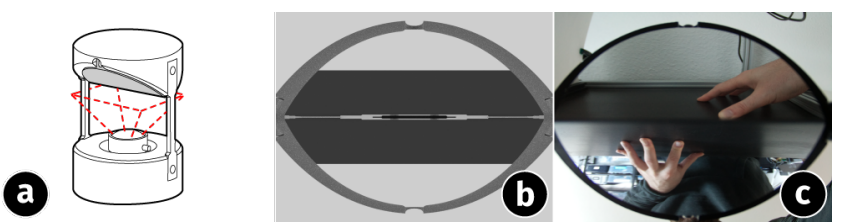

Figure 12: a) A camera is facing a bent mirror with two flat sides at 135 degrees. b) The simulated view of the camera facing upwards (red frustum in a). In the center, the reflection of the table surface, and space above the surface on both front and back of the tangible can be seen. c) Camera view of the manufactured prototype using the laminated PS mirror.

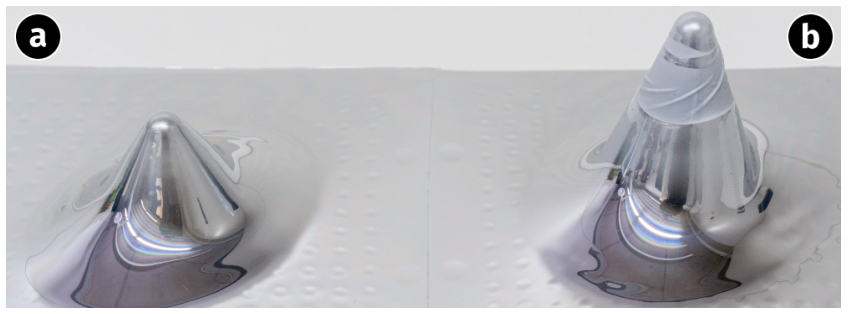

Figure 13: Stress test to achieve rupturing of the metallization layer during vacuum forming. a) $40 \times 50 \mathrm{~mm}$ cone b) $60 \times 50 \mathrm{~mm}$ cone.

\section{Tabletop Camera}

The tabletop camera is a tangible using a camera to track objects on the surface of a table. To widen the view of the camera and make use of only a single camera to track objects on both sides of the tangible, a mirror is used. Since this setup requires only two planar faces, no vacuum forming is necessary and a bent mirror is sufficient. The distance and angle can be evaluated using the MirrorForge simulation tool (see fig. 12) before carving the bents, cutting the outline, and bending the mirror on its 3D-printed fixture (see fig. 9). The tangible makes use of a Raspberry Pi HQ camera module (sensor: Sony IMX477, 12MP) with a $3 \mathrm{~mm}$ wide-angle lens to cover the mirror at only $45 \mathrm{~mm}$ distance. The mirror quality is sufficient to not affect the camera image at full resolution.

\section{DISCUSSION AND FUTURE WORK}

The proposed techniques based on $1 \mathrm{~mm}$ laminated PS sheets are especially suitable for small mirrors of up to $10-15 \mathrm{~cm}$ size and low curvatures. Larger sheets tend to bend and require additional fixtures. In addition to that, larger curvatures introduce a higher amount of material stretching which reduces dimensional accuracy towards high spots of the mirror shape. Even though stretching poses an issue to accuracy, the material allows strong deformation before the metallization layer breaks (see fig. 13). Reflection quality and distortion are suitable for prototypes that do not require sub-millimeter precision in the reflected image. Also, if mirror geometries are required containing both non-planar faces and sharp contours, none of the presented techniques is suitable. The main limitation of the presented simulation process is the decision to 
not consider projector or camera focus. This is not an issue with laser projectors or wide-angle cameras as shown in the application examples. However, when DLP projectors or camera lenses with a narrow focal plane are used, mirror geometries with large magnification factors may result in out-of-focus regions that can not be detected at the simulation step prior to fabrication. In the future, we plan to to extend the MirrorForge plugin to allowing live modification of the underlying surface equations of mirrors and give real-time simulation feedback. This would allow interactive control over the reflected image for enabling quick and easy exploration of suitable mirror geometries. In addition to the presented application examples, we can envision making use of the remaining flexibility in the thermoformed material in combination with hinged fixtures. This could allow the simple design and manufacturing of partially deformable mirrors in interactive education (teaching optics and astronomy) or art installations (projection mapping).

\section{CONCLUSION}

The presented techniques for bent, curved and freeform mirrors are suitable to create fast and low-cost prototypes with minimal tooling requirements. By using laminated polystyrene sheets mirror surfaces with complex shapes can be fabricated without an additional coating step. Depending on the application, these mirrors are either suitable for a fast evaluation of mirror calculations or as functional parts in prototypes. Reflection quality is sufficient for camera and short-throw projector applications as shown by the tangible tabletop camera and the circular projector. Fabrication relies only on a 3D printer and basic tooling, such as an improvised vacuum former or hand tools. Given this technique and the supporting toolchain for mirror design and simulation, the cost and time to produce prototypes with functional mirrors for HCI research can be drastically reduced.

\section{REPRODUCTION NOTE}

The application, source code, and 3D models are available publicly: https://github.com/volzotan/MirrorForge

\section{ACKNOWLEDGMENTS}

This work was funded by the Deutsche Forschungsgemeinschaft (DFG, German Research Foundation) through project EC437/1-1. We would like to thank Henning Pohl for discussions and feedback.

\section{REFERENCES}

[1] Karan Ahuja, Chris Harrison, Mayank Goel, and Robert Xiao. 2019. MeCap: Whole-Body Digitization for Low-Cost VR/AR Headsets. In Proceedings of the 32nd Annual ACM Symposium on User Interface Software and Technology (New Orleans, LA, USA) (UIST '19). Association for Computing Machinery, New York, NY, USA, 453-462. https://doi.org/10.1145/3332165.3347889

[2] Paul Bourke. 2005. Spherical Mirror: A New Approach to Hemispherical Dome Projection. In Proceedings of the 3rd International Conference on Computer Graphics and Interactive Techniques in Australasia and South East Asia (Dunedin, New Zealand) (GRAPHITE '05). Association for Computing Machinery, New York, NY, USA, 281-284. https://doi.org/10.1145/1101389.1101445

[3] Javaan Singh Chahl and Mandyam Veerambudi Srinivasan. 1997. Reflective surfaces for panoramic imaging. Appl. Opt. 36, 31 (Nov 1997), 8275-8285. https: //doi.org/10.1364/AO.36.008275

[4] Florian Echtler, Manuel Huber, and Gudrun Klinker. 2008. Shadow tracking on multi-touch tables. In AVI '08: Proceedings of the working conference on Advanced Visual Interfaces (Napoli, Italy). ACM, New York, NY, USA, 388-391. https: //doi.org/10.1145/1385569.1385640
[5] Otmar Hilliges, David Kim, Shahram Izadi, Malte Weiss, and Andrew Wilson. 2012. HoloDesk: Direct 3d Interactions with a Situated See-through Display. In Proceedings of the SIGCHI Conference on Human Factors in Computing Systems (Austin, Texas, USA) (CHI '12). Association for Computing Machinery, New York, NY, USA, 2421-2430. https://doi.org/10.1145/2207676.2208405

[6] Christoph Hölscher, Alexander Schnee, Hansjürgen Dahmen, Lokesh Setia, and Hanspeter A. Mallot. 2005. Rats are able to navigate in virtual environments. Journal of Experimental Biology 208, 3 (2005), 561-569. https://doi.org/10.1242/ jeb. 01371

[7] Hiroshi Ishiguro. 2001. Development of Low-Cost Compact Omnidirectional Vision Sensors. Springer New York, New York, NY, 23-38. https://doi.org/10.1007/9781-4757-3482-9 3

[8] Hideki Koike, Yoichi Sato, and Yoshinori Kobayashi. 2001. Integrating Paper and Digital Information on EnhancedDesk: A Method for Realtime Finger Tracking on an Augmented Desk System. ACM Trans. Comput.-Hum. Interact. 8, 4 (Dec. 2001), 307-322. https://doi.org/10.1145/504704.504706

[9] Rong-Hao Liang, Chao Shen, Yu-Chien Chan, Guan-Ting Chou, Liwei Chan, De-Nian Yang, Mike Y. Chen, and Bing-Yu Chen. 2015. WonderLens: Optical Lenses and Mirrors for Tangible Interactions on Printed Paper. Association for Computing Machinery, New York, NY, USA, 1281-1284. https://dl.acm.org/doi/ 10.1145/2702123.2702434

[10] Stefanie Mueller, Bastian Kruck, and Patrick Baudisch. 2013. LaserOrigami: Laser-Cutting 3D Objects. In Proceedings of the SIGCHI Conference on Human Factors in Computing Systems (Paris, France) (CHI '13). Association for Computing Machinery, New York, NY, USA, 2585-2592. https://doi.org/10.1145/2470654. 2481358

[11] Stefanie Mueller, Anna Seufert, Huaishu Peng, Robert Kovacs, Kevin Reuss, François Guimbretière, and Patrick Baudisch. 2019. FormFab: Continuous Interactive Fabrication. In Proceedings of the Thirteenth International Conference on Tangible, Embedded, and Embodied Interaction (Tempe, Arizona, USA) (TEI '19). Association for Computing Machinery, New York, NY, USA, 315-323. https://doi.org/10.1145/3294109.3295620

[12] Jörg Müller, Dieter Eberle, and Constantin Schmidt. 2015. BaseLase: An Interactive Focus+Context Laser Floor. In Proceedings of the 33rd Annual ACM Conference on Human Factors in Computing Systems (Seoul, Republic of Korea) (CHI '15). Association for Computing Machinery, New York, NY, USA, 3869-3878. https://doi.org/10.1145/2702123.2702246

[13] Claudio Pinhanez. 2001. The Everywhere Displays Projector: A Device to Create Ubiquitous Graphical Interfaces. In Ubicomp 2001: Ubiquitous Computing, Gregory D. Abowd, Barry Brumitt, and Steven Shafer (Eds.). Springer Berlin Heidelberg, Berlin, Heidelberg, 315-331. https://doi.org/10.1007/3-540-45427-6_27

[14] Michal Piovarči, Michael Wessely, Michał Jagielski, Marc Alexa, Wojciech Matusik, and Piotr Didyk. 2017. Directional Screens. In Proceedings of the 1st Annual ACM Symposium on Computational Fabrication (Cambridge, Massachusetts) (SCF '17). Association for Computing Machinery, New York, NY, USA, Article 1, 10 pages. https://doi.org/10.1145/3083157.3083162

[15] Christian Schüller, Daniele Panozzo, Anselm Grundhöfer, Henning Zimmer, Evgeni Sorkine, and Olga Sorkine-Hornung. 2016. Computational Thermoforming. ACM Trans. Graph. 35, 4, Article 43 (July 2016), 9 pages. https: //doi.org/10.1145/2897824.2925914

[16] Brygg Ullmer and Hiroshi Ishii. 1997. The MetaDESK: Models and Prototypes for Tangible User Interfaces. In Proceedings of the 10th Annual ACM Symposium on User Interface Software and Technology (Banff, Alberta, Canada) (UIST '97). Association for Computing Machinery, New York, NY, USA, 223-232. https: //doi.org/10.1145/263407.263551

[17] Nina Vaidya and Olav Solgaard. 2018. 3D printed optics with nanometer scale surface roughness. Microsystems \& Nanoengineering 4, 1 (July 2018), 1-8. https: //doi.org/10.1038/s41378-018-0015-4

[18] Andrew D. Wilson. 2005. PlayAnywhere: A Compact Interactive Tabletop Projection-Vision System. In Proceedings of the 18th Annual ACM Symposium on User Interface Software and Technology (Seattle, WA, USA) (UIST '05). Association for Computing Machinery, New York, NY, USA, 83-92. https://doi.org/10.1145/ 1095034.1095047

[19] Christian Winkler, Markus Löchtefeld, David Dobbelstein, Antonio Krüger, and Enrico Rukzio. 2014. SurfacePhone: A Mobile Projection Device for Singleand Multiuser Everywhere Tabletop Interaction. In Proceedings of the SIGCHI Conference on Human Factors in Computing Systems (Toronto, Ontario, Canada) (CHI '14). Association for Computing Machinery, New York, NY, USA, 3513-3522. https://doi.org/10.1145/2556288.2557075

[20] Bodo Wojakowski, Ulrich Klug, Jan Düsing, and Rainer Kling. 2010. Fabrication of thermoformable circuits by laser patterning of metallized thermoplastic foils. Physics Procedia 5 (2010), 301-309. https://doi.org/10.1016/j.phpro.2010.08.150

[21] Junichi Yamaoka and Yasuaki Kakehi. 2017. ProtoMold: An Interactive Vacuum Forming System for Rapid Prototyping. In Proceedings of the 2017 CHI Conference on Human Factors in Computing Systems (Denver, Colorado, USA) (CHI '17). Association for Computing Machinery, New York, NY, USA, 2106-2115. https: //doi.org/10.1145/3025453.3025498 